Part of Journal of Research of the National Bureau of Standards, Volume 33, July 1944

\title{
DIPOLE MOMENT AND STRUCTURE OF TRIOXANE
}

\author{
By Arthur A. Maryott and S. F. Acree
}

\section{ABSTRACT}

Trioxane is a solid polymer of formaldehyde resembling the well-known solvent dioxane in its chemical properties. The molecule can possibly exist in two spacially different forms resembling a chair and a cradle, for which the theoretical dipole moments are 2.3 and $0.6 \times 10^{-18}$ electrostatic unit (esu), respectively. The experimental value in benzene was found to be $2.18 \times 10^{-18}$ esu. Hence it is concluded that ordinary trioxane is largely in the chair form, possibly in equilibrium with a small amount of the cradle form. This cyclic ether would therefore be suitable for mixing with nonpolar solvents to study the effect of increased polarity on the activities and absorption spectra of indicators.

\section{CONTENTS}

I. Introduction

II. Experimental methods and results

III. Discussion

IV. References

\section{INTRODUCTION}

Trioxane, frequently called $\alpha$-trioxymethylene, is a cyclic trimer of formaldehyde. Its physical and chemical properties have been discussed by Walker and Carlisle [1]. ${ }^{1}$ Studies of Raman spectra [2] and crystal structure [3] indicate that it has a puckered, six-membered ring structure analogous to that of cyclohexane. Consequently, it may exist in two stereo isomeric forms, as discussed by Sachse [4] and by Mohr [5]. They are usually designated as the "chair," or Z, and the "cradle," or C forms.

Until recently the structure of cyclohexane and other six-membered monocyclic compounds was open to doubt. Chemical evidence based upon the Thorpe-Ingold hypothesis of valency deflection [6] indicated considerable strain in the cyclohexane ring and suggested a planar structure. On the other hand, the Sachse-Mohr theory of strainless rings required that these cyclic compounds have a puckered or multiplanar configuration and may exist in at least two isomeric forms. The isolation of two stable forms of decahydronaphthalene [7] corresponding to the "chair" and "cradle" structures confirmed the Sachse-Mohr theory for bicyclic compounds. Since then such isomerism has been demonstrated in a number of other bicyclic and polycyclic compounds. However, despite numerous attempts to isolate two such forms of cyclohexane derivatives and other mono-

${ }^{1}$ Figures $1 \mathrm{n}$ brackets indicate the literature references at the end of this article. 
cyclic compounds, there is no authentic proof of the separate existence of any such isomeric forms. Apparently, the strain of valency angles involved in passing from one of the strainless forms to the other is not sufficiently great to prevent rapid conversion of one to the other. Stability of the two isomers of decahydronaphthalene is supplied by the interlocking ring system, which prevents change of the two forms into each other without rupture of a covalent bond. One might expect that under ordinary conditions many six-membered monocyclic compounds would exist as equilibrium mixtures of the "chair" and "cradle" forms. It appears, however, that such a mixture containing appreciable amounts of both forms has been established only in the case of cyclohexane-1,4-dione.

The dipole moment of this compound in benzene solution has been found to be $1.3 D^{2}$ [8]. This value is intermediate between that calculated for the "chair" $(\mu=0)$ and the "cradle" $(\mu=3.9 D)$ forms and corresponds to an equilibrium mixture containing about 10 percent of the "cradle" form. In the case of heterocyclic compounds, such as dioxane, paraldehyde, and morpholine, as well as cyclohexane and its derivatives, definite evidence for the existence of the "chair" form alone has been obtained. The isolation of two forms of methylcyclohexane corresponding to the two possible multiplanar structures has been claimed recently [9] but conclusive proof is lacking.

To determine whether the "chair" or the "cradle" form is the stable one in solution at ordinary temperatures or whether the solution consists of an equilibrium mixture of the two forms, the dipole moment of trioxane has been measured using benzene as solvent. With trioxane, the "chair" form would have a fairly large dipole moment, whereas the "cradle" structure would have only a small moment. The experimental data give a dipole moment of $2.18 \mathrm{D}$ for trioxane.

\section{EXPERIMENTAL METHODS AND RESULTS}

Trioxane obtained from E. I. du Pont de Nemours \& Co. Inc., was recrystallized from cyclohexane and then sublimed. The melting point was $61^{\circ} \mathrm{C}$. The benzene, of analytical grade, was distilled immediately before use.

A heterodyne beat method was used for the measurement of the dielectric constants. The cell was a specially designed glass vessel with silver-mirror surfaces to serve as plates. Measurements were made in an air bath regulated at $30^{\circ} \pm 0.02^{\circ} \mathrm{C}$.

The experimental data are listed in table 1 . The molar polarization of the solute at infinite dilution, $P_{2}^{\infty}$, is calculated from the Hedestrand equation [10],

$$
P_{2}^{\infty}=\frac{3 P_{1}}{\left(\epsilon_{1}-1\right)\left(\epsilon_{1}+2\right)} \frac{\Delta \epsilon}{f_{2}}-\frac{P_{1}}{d_{1}} \frac{\Delta d}{f_{2}}+P_{1} \frac{M_{2}}{M_{1}}
$$

Wherever used, the subscripts 1,2 , and 12 refer to the pure solvent, solute, and solution, respectively, $f$ expresses the concentration in mole fraction, $\epsilon$ represents the dielectric constant, $d$ is the density, and $M$ is the molecular weight. $\Delta \epsilon$ equals $\epsilon_{12}-\epsilon_{1}$, and $\Delta d$ equals $d_{12}-d_{1} . P_{1}$, the molar polarization of the solvent, is defined as

${ }^{2} \mathrm{D}$ (Debye) $=10^{-18} \mathrm{esu}$. 


$$
\frac{\epsilon_{1}-1}{\epsilon_{1}+2} \frac{M_{1}}{d_{1}}
$$

The above equation reduces to

$$
P_{2}^{\infty}=14.86 \frac{\Delta \epsilon}{f_{2}}-30.67 \frac{\Delta d}{f_{2}}+0.3412 M_{2}
$$

when the numerical values for the constants of the solvent are inserted. The dielectric constant and density of pure benzene at $30^{\circ} \mathrm{C}$ were taken as 2.2627 and 0.8684 , respectively. $P_{E}$ is the electronic polarization obtained by summation of the atomic refractivities listed in Landolt-Bornstein Tabellen. The dipole moment was calculated from the relation

$$
\mu=0.223\left(P_{2}^{\infty}-P_{E}\right)^{\frac{1}{2}} \times 10^{-18} \text { esu. }
$$

TABLE 1.-Mole fraction, $f_{2}$, increment of dielectric constant, $\Delta \epsilon$, and dipole moment; $\mu$, of trioxane

\begin{tabular}{|c|c|}
\hline$f_{2}$ & $\Delta \epsilon$ \\
\cline { 1 - 2 } 0.00448 & 0.0276 \\
.01083 & .0671 \\
.01695 & .1054 \\
.02155 & .1342 \\
.02443 & .1516 \\
\hline & \\
\hline$\epsilon \epsilon / f_{2}=6.20$ & \\
$\Delta d / f_{2}=0.283$ & \\
$P_{2}^{\infty}=114.4$ & \\
$P_{K}=18.8$ \\
$\mu=2.18 \times 10^{-18} \mathrm{esu}$. \\
\hline
\end{tabular}

\section{DISCUSSION}

The two possible isomeric structures for trioxane are illustrated in figure 1. "The "chair" form has an axis of symmetry perpendicular to the plane of the three carbon atoms. The three oxygen atoms lie in a plane parallel to and slightly below this plane. Calculation of the dipole moment of the "chair" form by vector addition of the bond moments gives $2.3 \mathrm{D}$ if the $\mathrm{C}-\mathrm{O}$ and $\mathrm{C}-\mathrm{H}$ bond moments are taken as $0.9 \mathrm{D}$ and $0.2 \mathrm{D}$, respectively, and all of the valence angles are assumed to be tetrahedral. The moment calculated for the "cradle" form is $0.6 \mathrm{D}$. In view of the necessarily rather approximate nature of the calculated moment, the agreement between the actual value of $2.18 \mathrm{D}$ and that calculated for the "chair" form is as good as could be expected. The presence of a small amount of the practically nonpolar "cradle" form, however, is not excluded.

Trioxane is closely related structurally to the more familiar paraldehyde, which is a cyclic trimer of acetaldehyde containing alternate carbon and oxygen atoms in the ring and differing from trioxane only in that one hydrogen of each methylene group has been replaced by a methyl group. Unlike trioxane, however, which has only one possible structure for each of the two Sachse-Mohr forms, paraldehyde has four possible stereoisomeric structures for the "chair" and for the "cradle" configurations. Electron diffraction studies [11] of paraldehyde vapor are in favor of a "chair" structure, in which the 
three methyl groups are symmetrically located in a plane parallel to and close to the plane formed by the three carbon atoms of the ring, and in which all the valence angles are approximately tetrahedral. The dipole moment of paraldehyde has been found to be $2.03 D$ [12] in benzene solution. Although this value is somewhat lower than that found for trioxane, whereas a slightly higher value might be expected because of induction effects in the methyl groups, it does lend support to a "chair" structure for the molecule.

The cyclic ether, trioxane, in the molten state or mixed in various proportions with other solvents, would have very useful solvent properties somewhat similar to those of dioxane. It can be dissolved in varying amounts in nonpolar solvents to increase polarity, and in this connection should prove of interest in investigations on the effect of various polar and nonpolar organic compounds and solvents on the absorption spectra of indicators and their changes by organic acids and bases.

\section{REFERENCES}

[1] J. F. Walker and P. J. Carlisle, Chem. Eng. News, (Aug. 10, 1943).

[2] L. Kahovec and K. W. F. Kohlrausch, Z. physik. Chem. [B] 35, 29 (1937).

[3] N. F. Moerman, Rec. trav. chim. 56, 161 (1937).

[4] H. Sachse, Ber. deut. chem. Ges. 23, 1363 (1890).

[5] E. Mohr, J. prakt. Chem. 98, 315 (1918).

[6] J. F. Thorpe, J. Chem. Soc. 1020 (1931).

[7] W. Hückel, Liebigs Ann. Chem. 441, 1 (1925).

[8] C. G. Le Fevre and R. J. W. Le Fevre, J. Chem. Soc. 1696 (1935).

[9] D. M. Cowan, G. H. Jeffery, and A. I. Vogel, J. Chem. Soc. 1862 (1939).

[10] G. Hedestrand, Z. physik. Chem. [B] 2, 428 (1929).

[11] D. C. Carpenter and L. O. Brockway, J. Am. Chem. Soc. 58, 1270 (1936); P. G. Ackermann and J. E. Mayer, J. Chem. Phys. 4, 377 (1936).

[12] R. J. W. Le Fevre and P. Russell, J. Chem. Soc. 496, (1936).

Washington, April 22, 1944. 


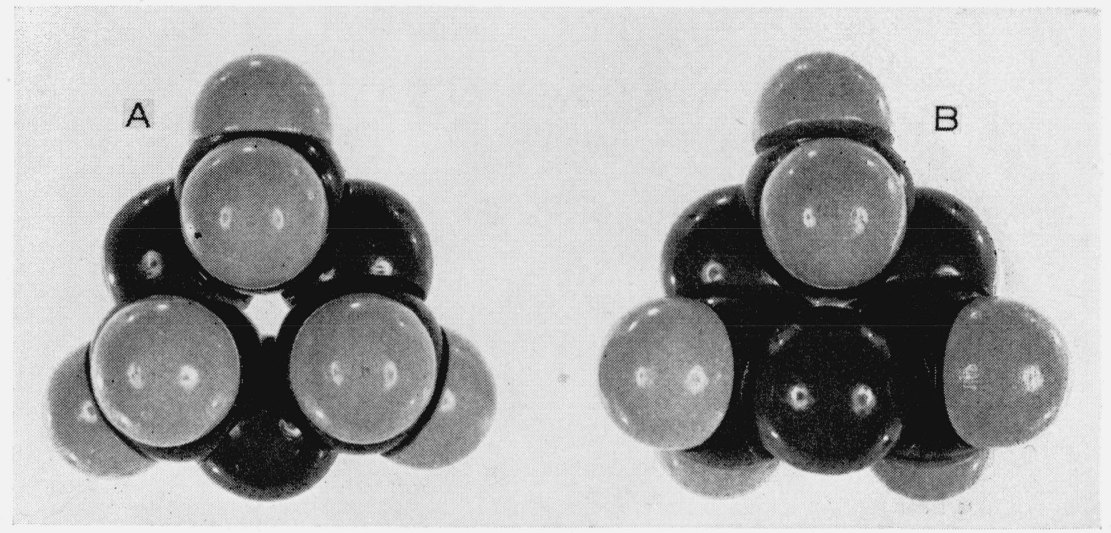

FIGURE 1.-Chair (A) and cradle $(B)$ configurations of trioxane.

Two hydrogens (light colored atom models) are attached to each earbon (black models). The other atom models are the oxygens connecting the earbons. 
NATIONAL BUREAU OF STANDARDS, WASHINGTON 25, D. C.

Send me the Mathematical Tables marked $\mathrm{X}$ below. I enclose remittance ${ }^{1}$ to cover the cost.

\begin{tabular}{|c|c|c|c|c|}
\hline Mark X & Title of publication & $\begin{array}{c}\text { United States and } \\
\text { its possessions, } \\
\text { and countries ex- } \\
\text { tending franking } \\
\text { privilege } \\
\end{array}$ & $\begin{array}{l}\text { Other } \\
\text { countries }\end{array}$ & Amount enclosed \\
\hline (1) & 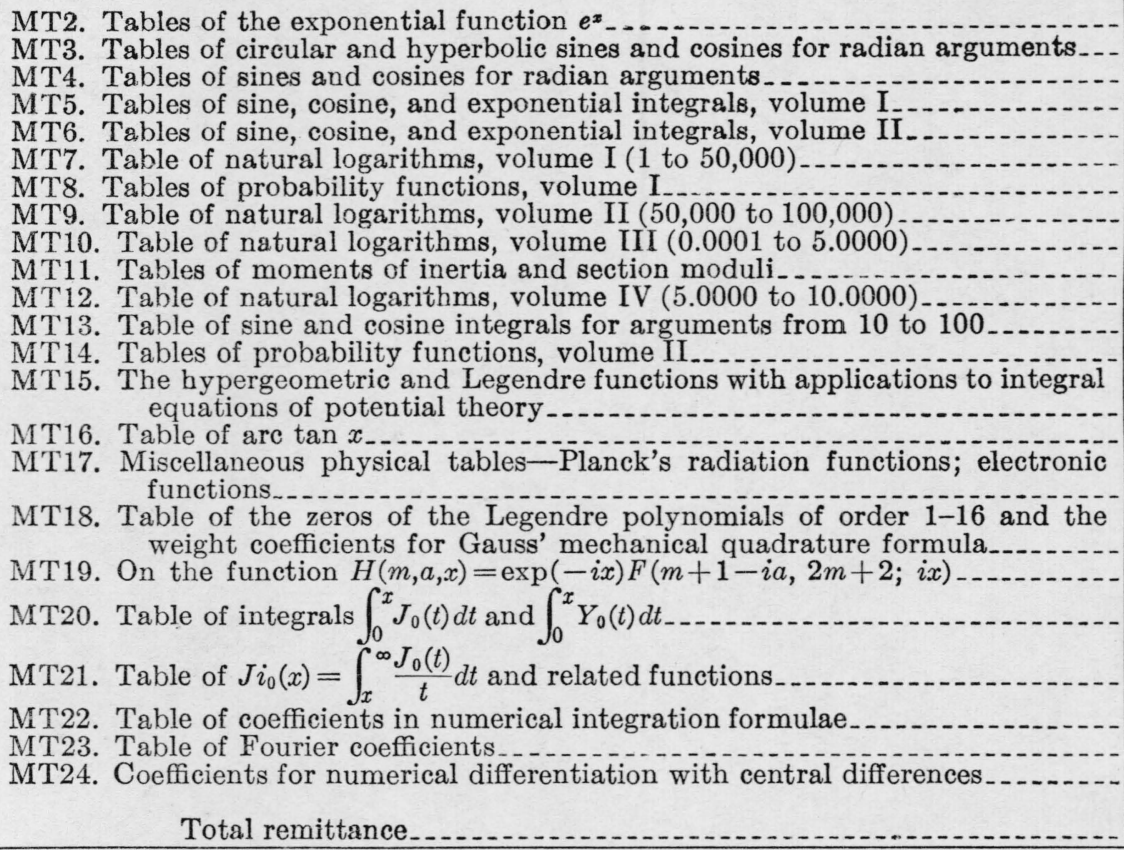 & $\begin{array}{r}\$ 2.00 \\
2.00 \\
2.00 \\
2.00 \\
2.00 \\
2.00 \\
2.00 \\
2.00 \\
2.00 \\
2.00 \\
2.00 \\
2.00 \\
2.00 \\
2.00 \\
2.00 \\
1.50 \\
.25 \\
.25 \\
.25 \\
.25\end{array}$ & $\begin{array}{r}\$ 2.50 \\
2.50 \\
2.50 \\
2.50 \\
2.50 \\
2.50 \\
2.50 \\
2.50 \\
2.50 \\
2.50 \\
2.50 \\
2.50 \\
2.50 \\
2.50 \\
2.50 \\
1.75 \\
\\
.30 \\
.30 \\
.30 \\
.30 \\
.30 \\
.30 \\
.30\end{array}$ & \begin{tabular}{|c|} 
\\
\end{tabular} \\
\hline
\end{tabular}

1 Remittance should be in form of post-office money order, or check, and made payable to the order of the "National Bureau of Standards" in United States currency.

Send to

Number and Street

City, Zone, and State. 


\section{MATHEMATICAL TABLES}

Attention is invited to a series of publications prepared by the Project for the Computation of Mathematical Tables conducted by the Federal Works Agency, Work Projects Administration for the City of New York, under the sponsorship of the National Bureau of Standards. The tables which have been made available through the National Bureau of Standards are listed below.

There is included in this list a publication on the hypergeometric and Legendre functions (MT15), prepared by the Bureau.

MT1. Table of the First Ten Powers of the Integers From 1 to 1000:

(1938) VIII + 80 pages; heavy paper cover. Out of print.

MT2. Tables of the Exponential Function $e^{x}$ :

The ranges and intervals of the argument and the number of decimal places in the entries are given below:

$\begin{array}{ccc}\text { Range of } x & \text { Interval of } x & \text { Decimal sgiven } \\ -2.5000 \text { to } 1.0000 & 0.0001 & 18 \\ 1.0000 \text { to } 2.5000 & .0001 & 15 \\ 2.500 \text { to } 5.000 & .001 & 15 \\ 5.00 \text { to } 10.00 & .01 & 12\end{array}$

(1939) XV+535 pages; bound in buckram, $\$ 2.00$.

MT3. Tables of Circular and Hyperbolic Sines and Cosings for Radian Arguments:

Contains 9 decimal place values of $\sin x, \cos x, \sinh x$ and $\cosh x$ for $x$ (in radians) ranging from

0 to 2 at intervals of 0.0001 .

(1939) XVII + 405 pages; bound in buckram, $\$ 2.00$.

MT4. Tables of Sines and Cosines for Radian Arguments:

Contains 8 decimal place values of sines and cosines for radian arguments ranging from 0 to 25 at intervals of 0.001 .

(1940) XXIX + 275 pages; bound in buckram, \$2.00.

MT5. Tables of Sine, Cosine, and Exponential Integrals, Volume I:

Values of these functions to 9 places of decimals from 0 to 2 at intervals of 0.0001 .

(1940) XXVI+444 pages; bound in buckram, $\$ 2.00$.

MT6. Tables of Sine, Cosine, and Exponential Integrals, Volume II:

Values of these functions to 9,10 , or 11 significant figures from 0 to 10 at intervals of 0.001 with auxiliary tables.

(1940) XXXVII + 225 pages; bound in buckram, $\$ 2.00$.

MT7. Table of Natural Logarithms, Volume I:

Logarithms of the integers from 1 to 50,000 to 16 places of decimals.

(1941) XVIII+501 pages; bound in buckram, $\$ 2.00$.

MT8. Tables of Probability Functions, Volume I:

Values of these functions to 15 places of decimals from 0 to 1 at intervals of 0.0001 and from

1 to 5.6 at intervals of 0.001 .

(1941) XXVIII+302 pages; bound in buckram, $\$ 2.00$.

MT9. Table of Natural Logarithms, Volume II:

Logarithms of the integers from 50,000 to 100,000 to 16 places of decimals.

(1941) XVIII + 501 pages; bound in buckram, \$2.CO.

MT10. Table of Natural Logarithms, Volume III:

Logarithms of the decimal numbers from 0.0001 to 50000 , to 16 places of decimals.

(1941) XVIII + 501 pages; bound in buckram, $\$ 2.00$.

MT11. Tables of the Moments of Inertia and Section Moduli of Ordinary Angles,"Chan. nels, and Bulb Angles with Certain Plate Combinations:

(1941) XIII +197 pages; bound in green cloth. $\$ 2.00$.

MT12. Table of Natural Logarithms, Volume IV:

Logarithms of the decimal numbers from 5.0000 to 100000 , to 16 places of decimals.

(1941) XXII+506 pages; bound in buckram, $\$ 2.00$.

MT13. Table of Sine and Cosine Intrgrals for Arguments From 10 to 100:

(1942) XXXII+185 pages; bound in buckram, $\$ 2.00$.

MT14. Tables of Probability Functions, Volume II:

Values of these functions to 15 places of decimals from 0 to 1 at intervals of 0.0001 and from 1 to 7.8 at intervals of 0.001 .

(1942) XXI+344 pages; bound in buckram, $\$ 2.00$.

[Continued on p. 4 of cover] 
MT15. The hypergeometric and Legendre functions with applications to integral equations of potential theory. By Chester Snow, National Bureau of Standards. Reproduced from original handwritten manuscript.

(1942) VII+319 pages, bound in heavy paper cover. $\$ 2.00$.

MT16, Table of Arc Tan $x$ :

Table of inverse tangents for positive values of the angle in radians. Second central differences are included for all entries.

$$
\text { (1942) XXV +169 pages; bound in buckram, } \$ 2.00 \text {. }
$$

$$
x=[0(.001) 7(.01) 50(.1) 300(1) 2,000(10) 10,000 ; 12 D \rrbracket
$$

MT17. Miscellaneous Physical Tables:

Planck's radiation functions (Originally published in the Journal of the Optical Society of America, February 1940); and

Electronic functions.

(1941) VII + 58 pages; bound in buckram, $\$ 1.50$.

MT18. Table of the Zeros of the Legendre Polynomials of Order 1-16 and the Weight Coefficients for Gauss' Mechanical Quadrature Formula:

(Reprinted from Bul. Amer. Mathematical Society, October 1942.)

5 pages with cover. 25 cents.

MT19. On the Function $H(m, a, x)=\exp (-i x) F(m+1-i a, 2 m+2 ; i x)$; with table of the confluent hypergeometric function and its first derivative.

(Reprinted from J. Math. Phys., December 1942.) 20 pages, with cover, 25 cents.

MT20. Table of Integrals $\int_{0}^{s} J_{0}(t) d t$ and $\int_{0}^{x} \Upsilon_{0}(t) d t$ :

Values of the two integrals are given for $x=0(.01) 10$ to 10 decimal places. (Reprinted from J. Math. and Phys., May 1943.) 12 pages, with cover, 25 cents.

MT21. Table of $J i_{0}(x)=\int_{x}^{\infty} \frac{J_{0}(t)}{t} d t$ and Related Functions:

Table I: $J i_{0}(x)$ to 10 decimal places and $F(x)=J i_{0}(x)+\log _{e} 1 / 2 x$ to 12 decimal places for $x=0(.1) 3$ with even central differences of $F(x)$.

Table II: $J i_{0}(x)$ to 10 decimal places, for $x=3(.1) 10(1) 22$ with even central differences up to $x=100$.

Table III: "Reduced" derivatives of $F(x)$ for $x=10(1) 21$ and $n=0(1) 13$, to 12 decimal places.

(Reprinted from J. Math. Phys., June 1943.) 7 pages, with cover, 25 cents.

MT22. Table of Coefficients in Numerical Integration Formulae:

The values of $B^{(n)}{ }_{n}(1) / n$ ! and $B^{(n)}{ }_{n} / n$ ! where $B^{(n)}{ }_{n}(1)$ denotes the $n^{\text {th }}$ Bernoulli polynominal of the $n$th order for $x=1$ and $B^{(n)}{ }_{n}$ denotes the $n^{\text {th }}$ Bernoulli number of the $n^{\text {th }}$ order, were com. puted for $n=1,2, \ldots 20$. The quantities $B^{(n)}{ }_{n}(1) / n !$ are required in the Laplace formula of numerical integration employing forward differences, as well as in the Gregory formula. The quantities $B^{(n)} n / n$ ! are used in the Laplace formula employing backward differences.

(Reprinted from J. Math. Phys., June 1943.) 2 pages, with cover, 25 cents.

MT23. TABle of Fourier Coefricients.

Whenever $\phi(x)$ is a known polynomial whose degree does not exceed 10 , the present table of the functions

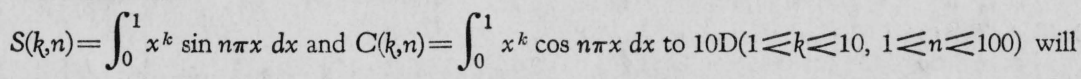
facilitate the evaluation of the first hundred Fourier Coefficients.

(Reprinted from J. Math. Phys. Sept., 1943.) 11 pages, with cover, 25 cents.

MT24. Coefricients for Numerical Difperentiation With Central Differences.

Coefficients are given for derivatives as far as the $52 \mathrm{~d}$. For the first 30 derivatives, exact values are given for coefficients of the first 30 differences, and also exact values are given for some coefficients of differences beyond the 30th. For the other coefficients, values are given to 18 significant figures.

(Reprinted from J. Math. Phys., Sept. 1943). 21 pages, with cover, 25 cents.

Payment is required in advance. Make remittance payable to the "National Bureau of Standards," and send with order, using the blank form facing page 3 of the cover.

A mailing list is maintained for those who desire to receive announcements regarding new tables as they become available. 